\title{
reviscafuences
}

ISSN: 1575.7072 | 8.15SN: 2172.7775

Páginas: 204-220

Recibido: 2020-06-15

Revisado: 2021-03-09

Aceptado: 2021-05-01

Preprint: 2021-05-10

Publicación Final: 2021-05-15

www.revistascientificas.us.es/index.php/fuentes/index

DOI: https://doi.org/10.12795/revistafuentes.2021.12052

\section{Inventario de Habilidades Socioemocionales y Salud Mental para Profesores de Educación Superior: validez de contenido}

\author{
Inventory of Socioemotional Skills and Mental Health for Professors in Higher \\ Education: validity of content
}

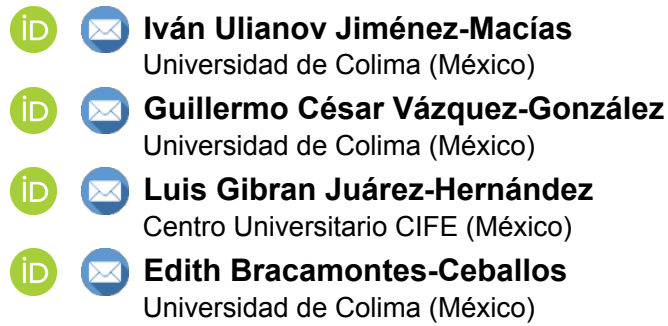

\section{Resumen}

Considerando que las habilidades socioemocionales y la salud mental tienen gran incidencia en todos los procesos educativos, se reporta la construcción de un instrumento de evaluación denominado Inventario de Habilidades Socioemocionales y Salud Mental para Profesores (IHSM-P) de educación superior. Se realizó un análisis documental para la definición de dos dimensiones, cada una con 40 ítems y seis áreas. Posterior a su construcción, el inventario fue sujeto de la revisión de cinco expertos, juicio de 20 expertos y pilotaje del instrumento con 21 docentes. A partir de la revisión de los expertos se realizó la mejora del instrumento y mediante el juicio de expertos se reveló la validez de contenido ( $V$ de Aiken $>0.80 ; \mid \mathrm{Cl}>0.75$ ), en términos de pertinencia y redacción de los ítems. A través del pilotaje se identificó buen grado de comprensión de instrucciones e ítems. Con fines orientativos se realizó el análisis de consistencia donde se obtuvo una adecuada confiabilidad (Alfa de Cronbach: 0.84). Se reconoce que la validez de contenido es una propiedad psicométrica relevante, sin embargo, se precisa la necesidad de ampliar la muestra para proseguir con el proceso de análisis de propiedades psicométricas, en específico confirmar la confiabilidad y analizar la propiedad de validez de constructo, la cual es referida como la principal de los tipos de validez.

\section{Abstract}

Considering that socioemotional skills and mental health have a high rate of incidence within all educational processes, the construction of an evaluation instrument it is offered, denominated Inventory of Socioemotional Skills and Mental Health for Professors (IHSM-P) of Higher Education. A documental analysis was performed for the definition of two dimensions, each one with 40 items and six areas. After its construction, the inventory was subject to the revision of five experts, the judgment of 20 experts, and a pilot trial of the instrument with 21 professors. After the revision of the experts, the improvement of the instrument was done and through the judgment of the experts it was revealed the validity of content (Aiken's V>0.80; $\mathrm{LCl}>0.75$ ), in terms of pertinence and writing of the items. Throughout the pilot trial, it was identified a good grade of comprehension of instructions and of the items. For orientation purposes, the consistency analysis was made, where good reliability was obtained (Cronbach's Alpha: 0.84 ). It is acknowledged that the validity of content is a relevant psychometric quality, although, the need to amplify the sample is required in order to proceed with the analysis process of the psychometric qualities, more specifically, to confirm the reliability and analyze the attribute of validity of construct, which is referred to as the main attribute of validity.

\section{Palabras clave}

salud mental, salud, profesor, habilidad, trastorno de la personalidad, instrumento de medida, inventario, instituto de 
enseñanza superior.

\section{Keywords}

mental health, health, teacher, skills, personality disorders, measuring instruments, inventories, higher education institutions.

\section{Introducción}

Evaluar las Habilidades Socioemocionales (HSE) y la Salud Mental (SM) permite a los profesores de educación superior, identificar, mantener y potenciar sus actividades docentes y funciones sustantivas, mediante el manejo de emociones propias, la resolución de conflictos y la toma de decisiones reflexivas, favoreciendo la empatía, la convivencia, cooperación y relaciones constructivas con los pares y generando un clima social y laboral positivo que impacta en el logro de metas personales, profesionales y en los objetivos académicos (Secretaría de Educación Pública, 2018). Cuando los profesores no reconocen sus HSE y SM presentan dificultad para resolver conflictos, desmotivación, desgaste, agotamiento, baja autoestima y autoconcepto, tensión emocional, ansiedad y depresión, así como diversos problemas somatomorfos (Arón \& Milicic, 2000; Martínez-Otero, 2003). El desequilibro o ausencia de uno de estos dificulta las actividades diarias, vida fructífera y productiva (Martínez, 2014).

La Organización Mundial de la Salud [OMS, 2013], en su Plan de acción sobre salud mental 2013-2020 menciona que, dependiendo del contexto cultural, económico y político, las personas desarrollan problemas de salud y trastornos mentales, además de la capacidad para la gestión de pensamientos, emociones, comportamientos e interacciones con los otros. Por su parte, las Naciones Unidas [ONU, 2015] con la intención de beneficiar a la sociedad actual y que las personas se desenvuelvan con potencialidad, dignidad e igualdad en un ambiente saludable, creó los Objetivos de Desarrollo Sostenible (ODS), planteando en el objetivo tres, la necesidad de garantizar la vida sana y promover el bienestar, mediante la prevención, el tratamiento y la promoción de la salud mental.

La SM es definida por la Organización Mundial de la Salud [OMS, 2017] como "un estado completo de bienestar físico, mental y social, y no solamente la ausencia de afecciones o enfermedades" (p.1); para el Instituto Mexicano del Seguro Social [IMSS, 2018], la SM "es el estado de equilibrio que debe existir entre las personas y el entorno socio-cultural que los rodea, incluye el bienestar emocional, psíquico y social e influye en cómo piensa, siente, actúa y reacciona una persona ante momentos de estrés" (párr.1), además de considerarla como la base para el bienestar y funcionamiento efectivo de los individuos y la comunidad. Tener buena SM implica saberse adaptar a las tensiones medioambientales, trabajar productivamente y con deseos de mejorar la condición social y personal (Kent, 2003).

Las HSE son un conjunto de capacidades que permiten gestionar sentimientos y emociones propias y de los demás, con la intención de guiar el pensamiento y acciones hacia un desempeño satisfactorio, lidiar de la mejor manera con distintos estados emocionales como una vía de motivación y sentido de vida (Elias, 2003; Mayer \& Salovey, 1997; Gobierno de México, 2013). La SM y las HSE resultan relevantes en el contexto educativo escolarizado, ante factores de riesgo como el tiempo de dedicación, la interacción y las múltiples exigencias administrativas; dichos factores, pueden ser medioambientales, biológicos, emocionales, cognitivos, conductuales, históricos, interpersonales o relacionados con los contextos donde se desarrolla el individuo, esencialmente los familiares y laborales (Cardozo-Gutiérrez, 2016).

La sociedad del conocimiento también ha generado cambios vertiginosos en todos los ámbitos de la vida social, provocando desafíos a los actores educativos (González \& Espinoza, 2018) y demandando del docente diferentes roles para la implementación de modelos emergentes y para el cumplimiento de actividades de gestión (Donoso, 2018). Las instancias internacionales han establecido compromisos para mitigar los efectos negativos y lograr un óptimo estado de salud mental, equilibrando habilidades cognitivas, sociales y afectivas que permitan el manejo de emociones (Organization for Economic Co-operation and Development [OECD, 2015]), especialmente en los profesores de educación superior, quienes participan en la formación de estudiantes que serán parte de una sociedad que exige interacción y comunicación, poniendo en juego actitudes, emociones, perspectivas y expectativas (Cardozo-Gutiérrez, 2016).

Una de las acciones de desarrollo de los recursos humanos que menciona la OMS para las universidades, es definir un componente de salud mental e incorporarlo en los programas de estudios de pregrado y posgrado (OMS 2013) como un medio para la formación, la calidad académica y calidad de vida de los profesores (Cassullo \& García, 2015; Ornelas \& Olivia, 2017).

Actualmente, existen herramientas para identificar la SM en adolescentes, jóvenes y adultos, evaluando la salud mental positiva, el estado de ánimo, el bienestar psicológico, actitudes, autoconocimiento, autoconcepto, crecimiento personal, bienestar subjetivo, calidad de vida, desarrollo positivo, funcionalidad, desesperanza, salud, salud en general, depresión, ansiedad y riesgo suicida (Aliaga et al., 2006; Beck \& Steer, 2011; Carrasco, 
Clemente \& Llanova, 1989; Castaños et al., 2011; García \& Vélez, 2017; Llach, 2004; Llunch, 1999; MartínLesende, 2013; Pierobon, 2020; Plutchik et al., 1989; Rubio et al.,1998; Sanz, 2014; Sanz \& Navarro, 2003; Sanz et al., 2003; Velarde-Jurado \& Ávila-Figueroa, 2002; Vilagut et al., 2005).

Las HSE se han evaluado mediante distintos instrumentos que identifican las habilidades emocionales y conductas socioadaptativas (Fernández-Berrocal et al., 2004), escalas de calificación conductual y emocional (Epstein, 2004), el desarrollo emocional (Pérez-Escoda et al., 2010), satisfacción laboral (Meliá \& Peiró, 1989, 1998a, 1998b), desgaste ocupacional (Uribe, 2006, 2007), estrategias y respuestas de afrontamiento (Cano et al., 2007; Mikulic \& Crespi, 2008 y Moss, 2010), la escala de habilidades sociales (Gismero, 2010), evaluación de habilidades blandas (McKenzie, 2014), inventarios de inteligencia emocional (Espinoza-Venegas et al., 2015), competencias socioemocionales (Mikulic, Crespi \& Radusky, 2015), la de habilidades socioemocionales y transversales (Huerta, 2019), así como la escala de diagnóstico de conducta adaptativa (Tassé et al., 2021); ninguno diseñado exprofeso para su aplicación en el contexto de la educación superior y exclusivamente para profesores.

Se registran otros instrumentos dirigidos a estudiantes con el nombre de habilidades sociales o socioemocionales que incorporan la observación del comportamiento cotidiano, mediante rúbricas (OECD, 2015), otros de evaluación no cognitiva, habilidades de pensamiento, toma de decisiones, socialización, motivación académica y perseverancia (Kafka, 2016), los que detectan habilidades sociales no cognitivas o blandas (Panizza, 2015; García, 2018), la evaluación de habilidades sociales y emocionales mediante informes, reportes, autoinformes, encuestas así como triangulación de métodos de evaluación (WilsonAhlstroma \& Yohalem, 2014; OECD, 2019; Kankaras et al., 2019, Kankaras \& Suarez-Álvarez, 2019; Caballo, 2000; Morán \& Olaz, 2014; Núñez et al., 2019), instrumentos que evalúan competencias y habilidades sociales y emocionales dirigidos a estudiantes y maestros de primaria y secundaria con la colaboración de diferentes países de Europa (Aguilar, et al., 2019); además, se ha encontrado evidencia de implementación de tecnología para la evaluación específica de HSE (Otero et al., 2017).

Recientemente, se encontraron en etapa de validación y pilotaje, cuestionarios de habilidades sociales y desempeño docente en IES (Araujo, 2017), así como de competencias socioemocionales percibidas en profesores universitarios (Llorent et al 2020). Evaluar las HSE conlleva complejidad para seleccionar, aplicar y construir instrumentos (Fragoso-Luzuriaga, 2015; Palomera et al., 2008), por lo que antecedentes teóricos y documentales como las aportaciones de García (2018), López-Mondéjar \& Tomás (2017) y OECD (2019), brindan sustento respecto a este tema. Es de relevancia insistir en que los instrumentos que sean construidos para la evaluación de las HSE deben ser válidos y confiables, considerando los principios recomendables en la construcción de instrumentos (Rikoon et al., 2016; McKown, 2017).

Por ende, el objetivo general fue construir y analizar la validez de contenido de un inventario que identifique la Salud Mental y las Habilidades Socioemocionales en los profesores de las IES, en consecuencia, los objetivos específicos son: 1) definir indicadores de SM y HSE a partir de la revisión teórica; 2) someter el instrumento a la revisión de expertos y juicio de expertos; 3 ) analizar la propiedad psicométrica de validez de contenido.

\section{Metodología}

Se trata de un estudio instrumental que consiste en el diseño, adaptación y análisis de propiedades psicométricas (Montero \& León, 2007). Para la construcción del instrumento se realizó una revisión teórica y análisis de cuestionarios afines (Tabla 1). A partir de lo anterior, se diseñó el Inventario de Habilidades Socioemocionales y Salud Mental para Profesores (IHSM-P) de educación superior el cual integró 76 ítems en 3 categorías: Habilidades Socioemocionales (HSE), Habilidades Socioemocionales-Salud Mental (HSE-SM) y Salud Mental (SM).

Tabla 1

Antecedentes

\begin{tabular}{|c|c|c|c|}
\hline No. & Instrumento & Autor & $\begin{array}{l}\text { Validez de contenido (Juicio de } \\
\text { expertos) }\end{array}$ \\
\hline 1 & $\begin{array}{l}\text { Inventario de Competencias } \\
\text { Socioemocionales para Adultos }\end{array}$ & $\begin{array}{l}\text { Mikulic, Crespi \& Radusky } \\
\text { (2015) }\end{array}$ & Sí \\
\hline 2 & $\begin{array}{l}\text { Inventario de respuestas de } \\
\text { afrontamiento para adultos }\end{array}$ & $\begin{array}{l}\text { Mikulic \&Crespi (2008) Moss } \\
(2010)\end{array}$ & No \\
\hline
\end{tabular}

Continued on next page 


\begin{tabular}{|c|c|c|c|}
\hline \multicolumn{4}{|c|}{ Table 1 continued } \\
\hline 3 & $\begin{array}{ll}\text { Cuestionario de } & \text { Desarrollo } \\
\text { Emocional de Adultos } & \end{array}$ & Pérez-Escoda et al. (2010) & Sí \\
\hline 4 & $\begin{array}{l}\text { Cuestionario de satisfacción } \\
\text { laboral }\end{array}$ & $\begin{array}{l}\text { Meliá y Peiró }(1989,1998 a \text {, } \\
\text { 1998b) }\end{array}$ & $\begin{array}{l}\text { *se apoya en el muestreo de ítems } \\
\text { de cuestionarios y de contenidos de } \\
\text { la legislación recogidos en el S4/82. }\end{array}$ \\
\hline 5 & $\begin{array}{l}\text { Escala mexicana de desgaste } \\
\text { ocupacional }\end{array}$ & Uribe (2006) Uribe (2007) & No \\
\hline 6 & $\begin{array}{l}\text { Inventario de Estrategias de } \\
\text { Afrontamiento }\end{array}$ & Cano et al. (2007) & No \\
\hline 7 & $\begin{array}{l}\text { Escala para la evaluación } \\
\text { de la expresión, manejo y } \\
\text { reconocimiento de emociones, y } \\
\text { escala de inteligencia emocional }\end{array}$ & $\begin{array}{l}\text { Fernández-Berrocal et al. } \\
\text { (2004) Espinoza-Venegas et } \\
\text { al. (2015) }\end{array}$ & Sí \\
\hline 8 & Escala de desesperanza de Beck & Aliaga et al. (2006) & No \\
\hline 9 & El Cuestionario de Salud SF-36 & Vilagut et al. (2005) & No \\
\hline 10 & Inventario de Ansiedad de Beck & $\begin{array}{l}\text { Sanz (2014); Sanz \& Navarro } \\
\text { (2003); Beck \& Steer (2011) }\end{array}$ & No \\
\hline 11 & $\begin{array}{l}\text { Inventario para la Depresión de } \\
\text { Beck-II }\end{array}$ & Sanz et al. (2003) & No \\
\hline 12 & $\begin{array}{l}\text { Escala de riesgo suicida de } \\
\text { Plutchik }\end{array}$ & $\begin{array}{l}\text { Rubio et al. (1998) Plutchik et } \\
\text { al. (1989) }\end{array}$ & No \\
\hline 13 & $\begin{array}{l}\text { Inventario de aserción de Gambrill } \\
\text { y Richey }\end{array}$ & $\begin{array}{l}\text { Carrasco et al. } \\
\text { Castaños et al. (2011) }\end{array}$ & No \\
\hline
\end{tabular}

Posterior a su construcción, el instrumento se sometió a revisión de cinco expertos quienes analizaron la pertinencia, calidad y claridad de la redacción de cada ítem y la relevancia de las categorías. La siguiente revisión se realizó mediante un juicio de 20 expertos del área educativa para la evaluación de la pertinencia y redacción de los ítems mediante un enfoque cuali-cuantitivo. Para la evaluación cuantitativa se empleó la escala de jueces expertos y para su análisis se usó el coeficiente de validez de contenido $V$ de Aiken y sus intervalos de confianza al 95\%, considerándose como valor mínimo de 0.80 para la aceptación de un ítem como válido (Penfiel y Giacobbi, 2004), y para el valor inferior del intervalo de confianza se especificó un valor mayor a 0.60. La selección de expertos y jueces siguió criterios formales y variables relevantes (Juárez-Hernández \& Tobón, 2018). (Tabla 2).

\section{Tabla 2}

Caracterización del perfil de expertos y competencia de los jueces

\begin{tabular}{|c|c|c|}
\hline $\begin{array}{l}\text { Características } \\
\text { Género: }\end{array}$ & $\begin{array}{l}\text { Expertos } \\
40 \% \text { Masculino } 60 \% \text { Femenino }\end{array}$ & $\begin{array}{l}\text { Jueces } \\
50 \% \text { Masculino } 50 \% \text { Femenino }\end{array}$ \\
\hline Roles: & Docentes: 5 Directivos: 1 & $\begin{array}{l}65 \% \text { Profesor por horas } 35 \% \text { Profesor- } \\
\text { investigador }\end{array}$ \\
\hline Grado académico: & Maestría: 4 Doctorado: 1 & Maestría: 10 Doctorado: 10 \\
\hline Edad: & $\bar{X}=35.33 . \sigma=8.36$ & $\bar{X}=37.50 . \sigma=10.24$ \\
\hline
\end{tabular}




\begin{tabular}{|c|c|c|}
\hline $\begin{array}{l}\text { Áreas de experiencia } \\
\text { profesional: }\end{array}$ & $\begin{array}{l}\text { Docencia, calidad de vida, formación, } \\
\text { procesos psicológicos, sociales, } \\
\text { organizacionales y salud. }\end{array}$ & $\begin{array}{l}\text { Docencia, calidad de vida, género, } \\
\text { formación, investigación, procesos } \\
\text { psicológicos, psicología, sociales, salud, } \\
\text { organizacionales, de violencia, filosofía, } \\
\text { comunicación y tecnología educativa. }\end{array}$ \\
\hline $\begin{array}{l}\text { Años de experiencia } \\
\text { profesional: }\end{array}$ & $\bar{X}=13$ & $\bar{X}=16$ \\
\hline $\begin{array}{l}\text { Artículos publicados en el } \\
\text { área: }\end{array}$ & $\bar{X}=9$ & $\bar{X}=7$ \\
\hline $\begin{array}{l}\text { Ponencias presentadas en el } \\
\text { área: }\end{array}$ & $\bar{X}=5$ & $\bar{X}=10$ \\
\hline $\begin{array}{l}\text { Capítulos de libro publicados } \\
\text { en el área: }\end{array}$ & $\bar{X}=2$ & $\bar{X}=5$ \\
\hline $\begin{array}{l}\text { Experiencia docente- } \\
\text { investigativa: } \\
\text { Experiencia en la revisión, } \\
\text { diseño o validación } \\
\text { de instrumentos de } \\
\text { investigación: }\end{array}$ & $\begin{array}{l}\bar{X}=9 \\
60 \%\end{array}$ & $\begin{array}{l}\bar{X}=10 \\
95 \%\end{array}$ \\
\hline
\end{tabular}

Finalmente, el inventario se aplicó a 21 docentes de IES estatales y privadas quienes contestaron 5 preguntas que pertenecen al cuestionario de satisfacción del instrumento (CIFE, 2018) para medir el nivel de comprensión de instrucciones, dimensiones, áreas e ítems. Como una fase de prueba y con fines orientativos se efectuó un análisis de consistencia interna mediante el cálculo del coeficiente Alfa de Cronbach (Cronbach, 1951) y su intervalo de confianza al 95\% con la propuesta de Koning y Franses (2003). Para el valor obtenido por el Alfa de Cronbach se consideró el valor mínimo aceptable de 0.70 , y los valores preferenciales entre 0.80 y 0.90 (Nunnally \&Bernstein, 1994; Juárez-Hernández, 2018).

\section{Resultados}

Después de la revisión teórica se identificó que, de los instrumentos localizados, tres presentan un proceso de validación de contenido (Meliá \& Peiró 1989, 1998a, 1998b; Mikulic et al., 2015; Pérez-Escoda et al., 2010) y cinco la revisión de expertos y validación de facie. En la categoría SM, hay ausencia de análisis de validez de contenido. Los instrumentos cuentan con datos sobre nivel de consistencia con Alfa de Cronbach y evalúan 3 aspectos de la HSE: la conciencia, la regulación y comunicación sin considerar la expresión, el ámbito profesional, laboral, autoconocimiento y el estado personal. Con relación a la SM se aborda de manera parcial la sintomatología de la desesperanza, la ansiedad, depresión y suicidio, pero su estructura no valora padecimientos, trastornos y problemáticas de salud, como ansiedad, depresión, desesperanza, asertividad, suicidio y salud en general (Céspedes, 2008; Alemañy, 2009; Alonso, 2014; Marenco-Escudero \& Ávila-Toscano, 2016; Crosier \& Donkova, 2018), así como su relación con las HSE.

Lo anterior, permitió definir la estructura del Inventario de Habilidades Socioemocionales y Salud Mental para Profesores de educación superior. En esta fase, se sustituyó el nombre de categoría por el de dimensión y el de subcategoría por área; se agruparon 76 ítems en dos dimensiones y se definieron tres áreas para cada una de ellas, además se incluyeron ítems para identificar el sentido de vida y el estrés, aspectos que no se encontraron en ninguno de los cuestionarios revisados. Con la revisión de expertos se presentó una nueva versión del instrumento. Se atendieron 12 sugerencias en las áreas, la estructura y redacción; se eliminaron cuatro ítems y se crearon dos, quedando un total de 80 ítems, dos dimensiones y seis áreas. A partir de esta revisión el 100 por ciento de los ítems se redactó en primera persona y con el verbo en infinitivo. (Tabla 3). 
Tabla 3

Modificaciones a la estructura del inventario.

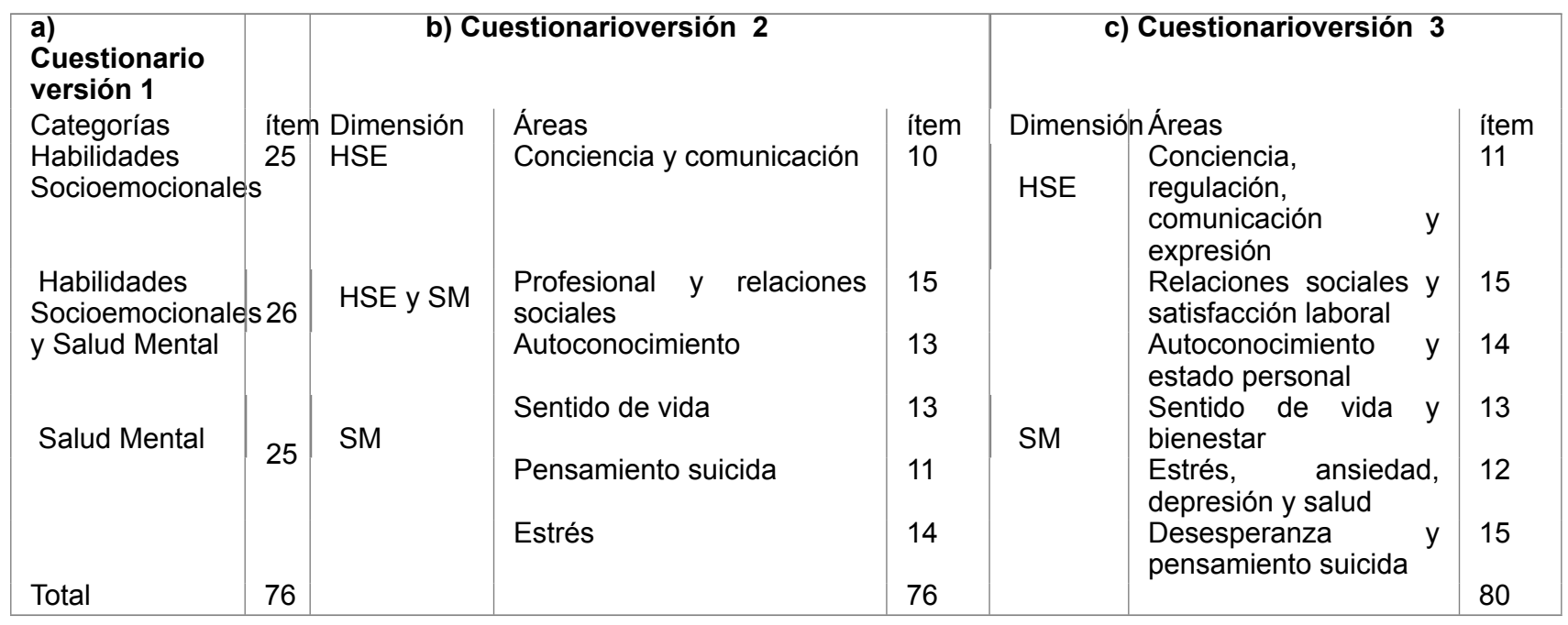

Con la evaluación cualitativa de los jueces expertos se indicaron sugerencias de mejora en la redacción de 21 ítems y su pertinencia a las áreas. Estas recomendaciones fueron relacionadas con el uso de signos de puntuación y se definió una sola acción en nueve de ellos. 
Por su parte, el análisis cuantitativo reveló la validez de contenido de los ítems propuestos en términos de pertinencia y redacción ( $\mathrm{V}$ de Aiken $>0.80 ; \mathrm{ICl}>0.75$ ). (Figura 1 y 2 ).

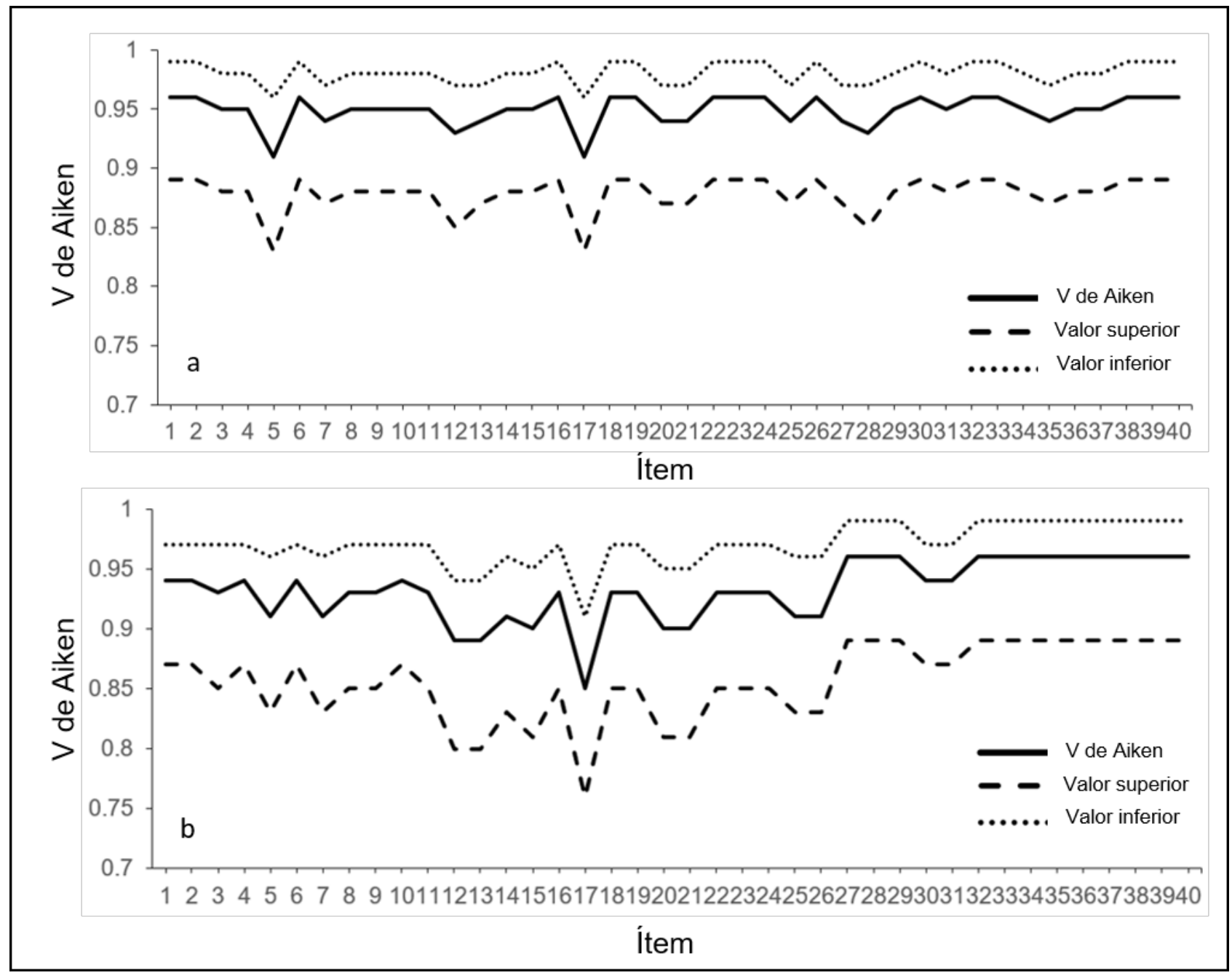

Figura 1. Índice de validez de contenido (V de Aiken) e intervalos de confianza al $95 \%$ para los criterios de pertinencia (a) y redacción (b) de los ítems de Habilidades Socioemocionales. 


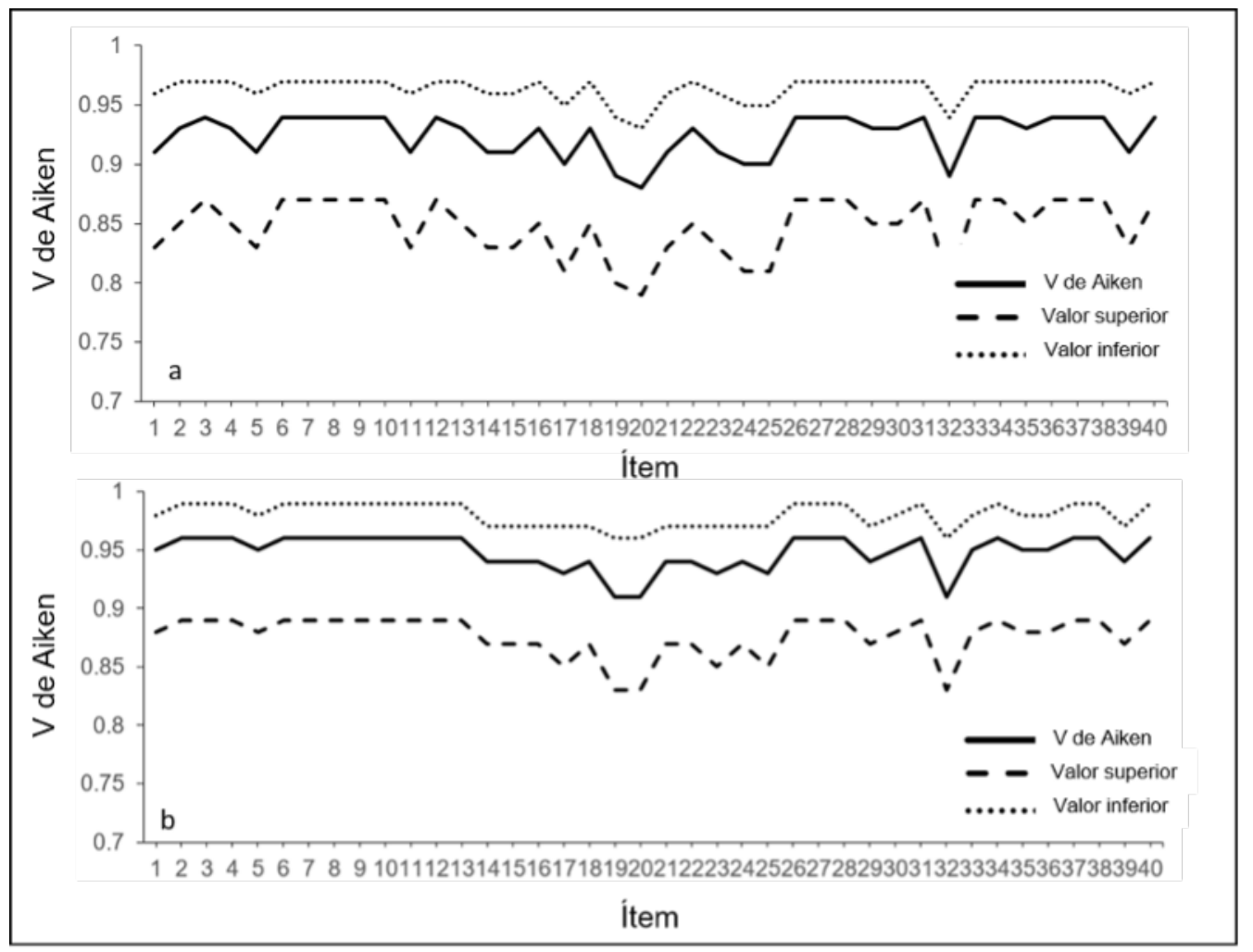

Figura 2. Índice de validez de contenido (V de Aiken) e intervalos de confianza al $95 \%$ para los criterios de pertinencia (a) y redacción (b) de los ítems de SM.

Derivado de lo anterior, el instrumento que a continuación se presenta consta de 80 ítems distribuidos en 2 dimensiones y 6 áreas. La escala psicométrica utilizada es tipo Likert de 5 opciones: a) nunca, b) casi nunca, c) algunas veces, d) casi siempre y e) siempre. Cada dimensión cuenta con igual número de ítems, la primera que analiza las habilidades socioemocionales y la otra, la salud mental. (Tabla 4).

\section{Tabla 4}

Instrumento generado a partir del juicio de expertos.

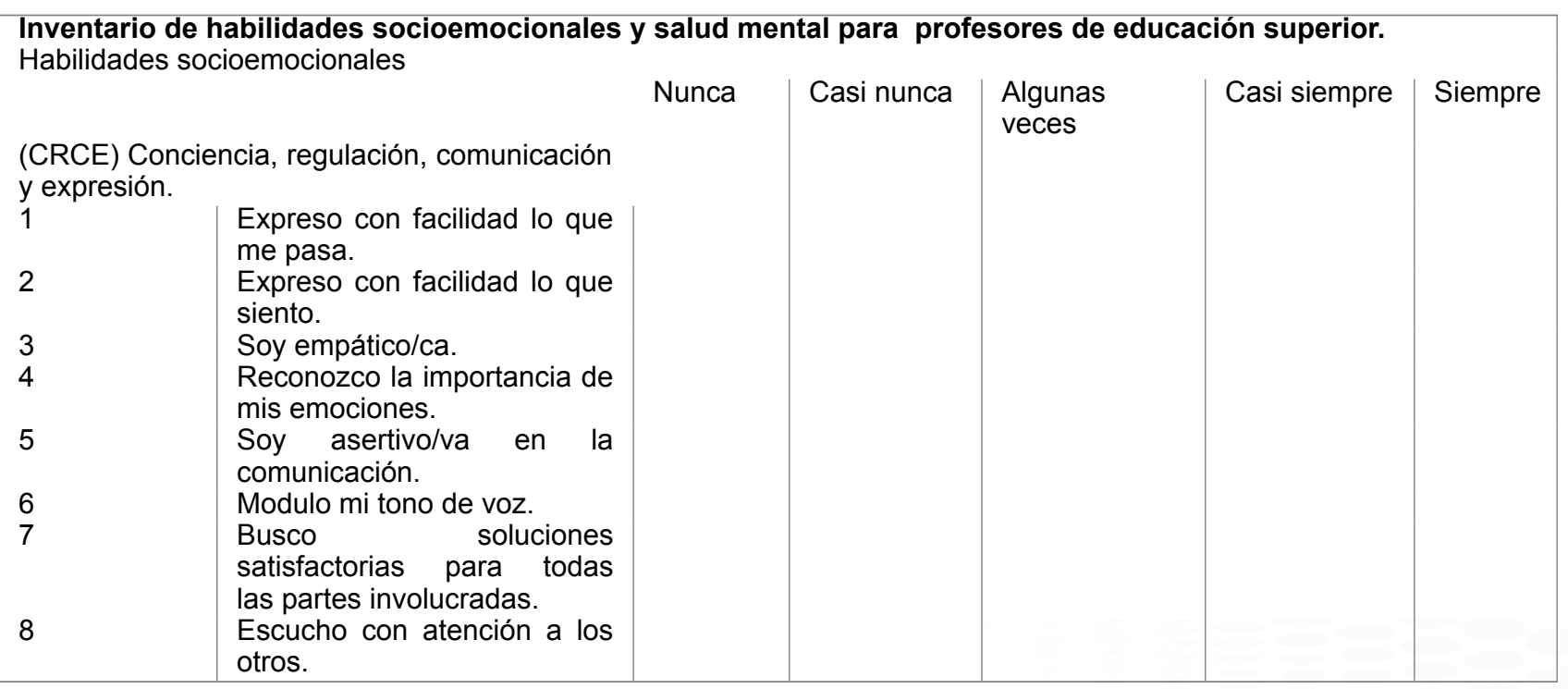




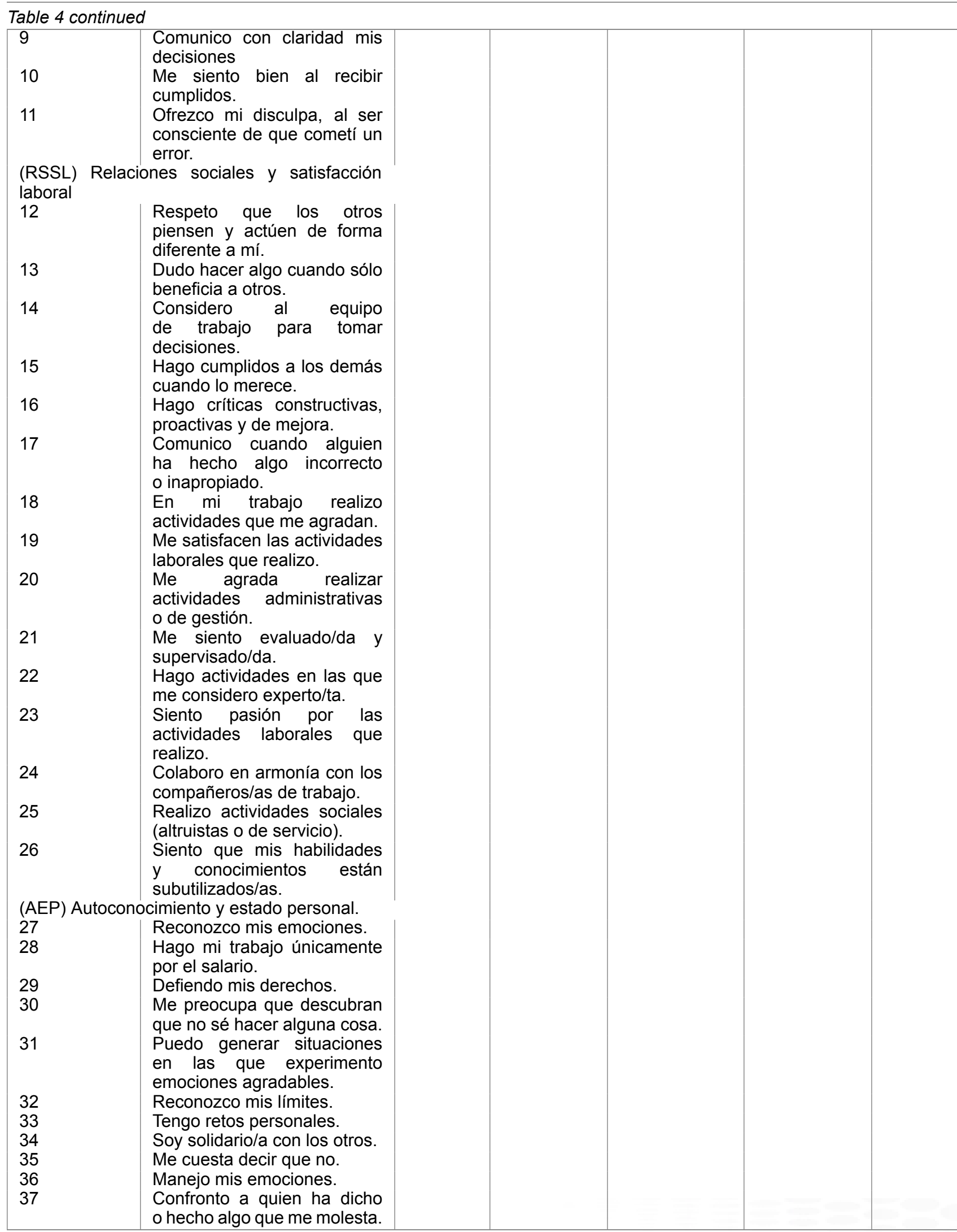




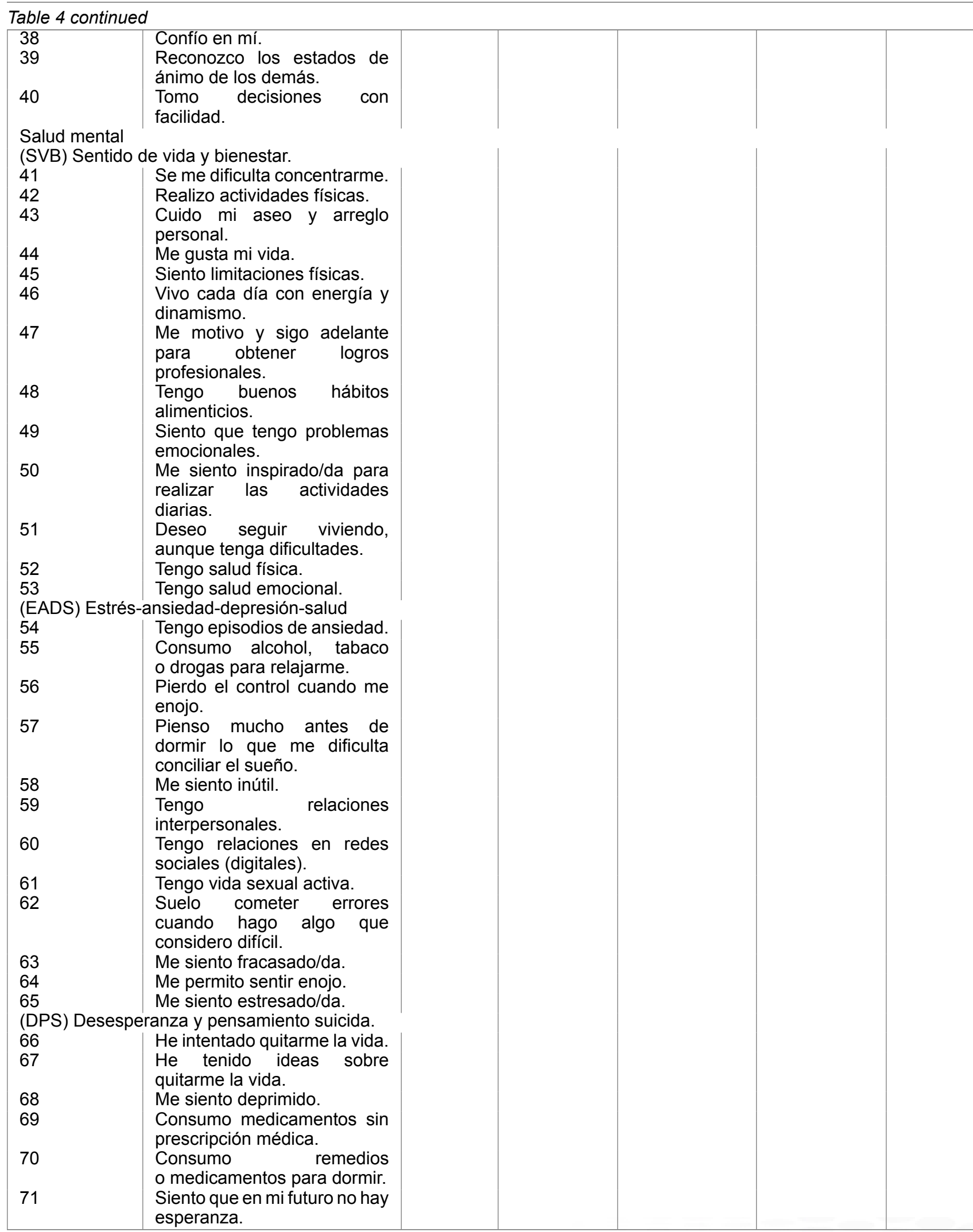




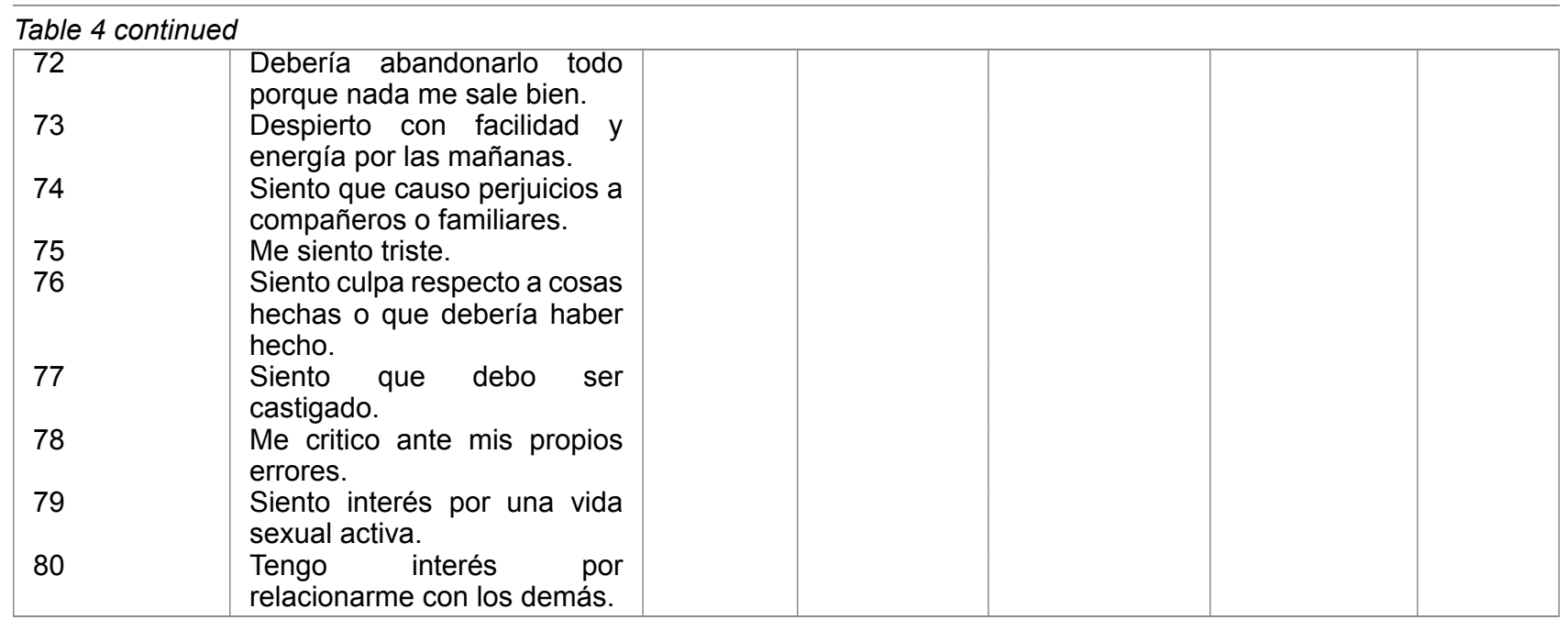

La encuesta de satisfacción para el inventario, por el grupo piloto, reveló que la comprensión del instrumento, instrucciones e ítems fue favorable. Respecto al análisis inicial de consistencia interna, se presentó un valor óptimo (Alfa de Cronbach: 0.84$)$ y acorde al intervalo de confianza al $95 \%(0.725+0.923)$, el valor inferior se encuentra dentro de lo estipulado como aceptable. (Tabla 5).

Tabla 5

Resultados de la encuesta de satisfacción para el inventario IHSM-P

\begin{tabular}{|c|c|c|c|c|c|}
\hline $\begin{array}{l}\text { Rubro } \\
\text { Comprensión de las instrucciones } \\
\text { Comprensión de los ítems } \\
\text { Nivel de satisfacción general con el inventario }\end{array}$ & $\begin{array}{l}\text { Malo } \\
0 \% \\
0 \% \\
0 \%\end{array}$ & $\begin{array}{l}\text { Regular } \\
0 \% \\
9.50 \% \\
0 \%\end{array}$ & $\begin{array}{l}\text { Bueno } \\
19 \% \\
4.80 \% \\
23.80 \%\end{array}$ & $\begin{array}{l}\text { Muy } \\
\text { bueno } \\
52 \% \\
57.10 \% \\
47.60 \%\end{array}$ & $\begin{array}{l}\text { Excelente } \\
28.60 \% \\
28.60 \% \\
8.60 \%\end{array}$ \\
\hline
\end{tabular}

\section{Discusión}

La lectura de los resultados señala en primer lugar la falta evidente de instrumentos específicos para valorar las habilidades socioemocionales y la salud mental de los profesores de educación superior; aun cuando se encontraron distintas escalas, inventarios y cuestionarios, ninguno responde de manera específica al constructo que aquí se estudia. La mayoría de los autores clasifican las escalas combinando distintas áreas, lo que complejiza su valoración; además, su análisis depende también del contexto y del momento histórico, teniendo diferentes denominaciones que van desde evaluar mediante cuestionario las habilidades sociales y desempeño docente en institutos de educación superior (Araujo, 2017), competencias socioemocionales percibidas en profesores universitarios (Llorent, Zych \& Varo-Millán, 2020), habilidades sociales (Caballo, 2000; Olaz, 2014; Núñez et al., 2019), competencias y habilidades sociales y emocionales (Aguilar, et al 2019), dirigidos a estudiantes y maestros de niveles educativos básicos, es decir, primaria y secundaria; para el caso de la salud mental se encuentran instrumentos que evalúan la funcionalidad (Martín-Lesende, 2013; Pierobon, 2020), salud (Llunch, 1999), salud en general, hasta calidad de vida (Velarde-Jurado \& Ávila-Figueroa, 2002; Llach, 2004; García \& Vélez, 2017).

En segundo lugar, de acuerdo con las investigaciones consultadas queda de manifiesto que las HSE aportan significativamente beneficios a los profesores y a su entorno (Jones et al., 2013), reconocerlas favorece la gestión de experiencias positivas y proporciona herramientas de paz (Rodríguez, 2017, Gutiérrez-Torres \& Buitrago-Velandia, 2019 que pueden generar impactos positivos en las actitudes, conductas, comportamientos, el desempeño, clima escolar y mejorar la percepción respecto a los centros educativos; la relación entre estudiantes y profesores, y favorecer el aumento de compromiso que promueve el aprendizaje cooperativo (Mena et al., 2009, Papieska et al., 2016; Schonert-Reichl, 2017; Whitehead \& Suave, 2018), por lo que identificar las HSE y la SM en los profesores a partir de instrumentos puede facilitar la promoción del bienestar y prevenir la frecuencia de problemas de salud mental en los sectores educativos derivados de brotes y trastornos como el estrés, ansiedad y depresión, así como el consumo de sustancias, la violencia en el trabajo, el acoso, la intimidación, el ausentismo, la desmotivación, la baja calidad y productividad laboral (Mingote \& Núñez, 
2011).

En tercer lugar, los resultados señalados coinciden con políticas nacionales e internacionales para enfocarse en la recuperación de datos empíricos en relación con la salud mental y las HSE de sus profesores y que podría servir de referencia a las IES, recalcando que, para transitar de las evidencias a las acciones, se deben definir indicadores básicos sobre salud mental con sus determinantes sociales y temporales (OMS 2013).

En cuarto lugar, se coincide con diversos autores que resaltan la importancia del análisis de las propiedades psicométricas de los instrumentos, por tal motivo, en el presente se estableció un marco metodológico de fases concatenadas con objetivos particulares para lograr un inventario que cumpla con criterios de calidad (Kerlinger \& Howard, 2002; Mendoza-Mendoza \& Baldemar, 2009), por lo que una de las fases es la validez de contenido que provee evidencia respecto al grado de relevancia y pertinencia de los elementos de un instrumento con relación al objetivo de constructo (Haynes et al., 1995)

Con el marco metodológico constituido por la revisión de expertos se verificó que los ítems son pertenecientes al constructo, su relevancia y su adecuada redacción y comprensión (Anastasi y Urbina, 2008; Buela-Casal y Sierra, 1997 y Kerlinger y Howard, 2002). Un aspecto para destacar es que un factor fundamental tanto para la revisión de expertos y juicio de expertos fue su selección y número de ellos (Fernández-Gómez et al., 2020; Juárez-Hernández \& Tobón, 2018; Navarro-Prado et al,. 2020; Vélez et al., 2021). En específico el juicio de expertos, aportó mejoras en la redacción de los ítems y la pertinencia a las áreas, con la participación de 20 jueces, un número mayor a lo que estipulan diversos autores (Hyrkäs et al., 2003; Mills et al., 2012). Referente al juicio de expertos y evaluación, el análisis cuantitativo mediante el cálculo de $V$ de Aiken y sus intervalos de confianza al $95 \%$, permitió precisar la validez de contenido de los ítems que se traduce como el grado de una medida representativa de los contenidos, así como el alcance de cada elemento o dimensión de constructo (Ruíz, 2002), confirmando que el instrumento es válido y representativo en términos de redacción y pertinencia. Para aquellos ítems cercanos al mínimo aceptado de 0.80 , si bien fueron validados, se revisaron también a partir de la valoración de los jueces. En relación con los resultados del grupo piloto, permitieron verificar la comprensión de la redacción de los ítems e instrucciones, así como el grado de satisfacción del cuestionario, aspectos importantes que pueden afectar las propiedades psicométricas de un instrumento (Haynes et al., 1995; Meliá, 2001).

Respecto al análisis inicial de consistencia interna, se obtuvo un valor óptimo (Alfa de Cronbach: 0.84), lo cual se traduce como el grado en que los ítems de un instrumento miden el mismo aspecto o lo que se desea medir, se verifica la exactitud de los ítems y la aplicación repetida produce los mismos resultados (Cardona et al., 2011; González-Ortega, 2008; Hernández-Sampieri et al., 2003), de la misma manera, refleja la correlación entre ítems y la representación del concepto abordado (Welch y Comer, 2001). Sin embargo, como lo refiere Charter (1999), el valor del coeficiente tiende a ser inestable con muestras pequeñas, lo cual se puede observar la oscilación obtenida mediante el intervalo de confianza al 95\%, por lo que el valor aquí obtenido únicamente tendrá que ser visto con un fin orientativo y no conclusivo.

Finalmente, es importante reconocer las limitaciones del alcance del instrumento aquí presentado, siendo la primera de ellas que el instrumento está dirigido a una población específica, que son profesores de educación superior. Por la trascendencia del tema se considera fundamental adaptar el instrumento a otros niveles educativos. La segunda, involucra lo referente al proceso de validación, ya que si bien el instrumento fue validado en facie y contenido, se requiere proseguir con el análisis de sus propiedades psicométricas, destacando la validez de constructo, la cual es considerada como la principal de los tipos de validez de acuerdo a la metodología para la construcción de instrumentos de medición en salud y que consiste en demostrar que el inventario funciona para lo que se creó mediante procesos estadísticos (Cruz-Avelar \& Cruz-Peralta, 2017), es decir, verifica si la estructura del cuestionario e ítems reproducen el constructo planteado (Mavrou, 2015; Lagunes-Córdoba, 2017).

\section{Conclusiones}

Con base en los resultados obtenidos se puede constatar que el Inventario de Habilidades Socioemocionales y Salud Mental para Profesores (IHSM-P) de educación superior está integrado por dos dimensiones con tres áreas cada una y un total de 80 ítems. El proceso de validación permitió la mejora constante de la redacción y adecuar la estructura del instrumento. El presente inventario denota que posee validez de contenido que se traduce como la representatividad en la definición del constructo, representatividad del grupo de ítems, aspectos gramaticales de los ítems y claridad de las instrucciones (Koller, Levenson y Glück, 2017). Se reconoce la necesidad de aplicar el instrumento a una muestra mayor y representativa para proseguir con el análisis de las propiedades psicométricas. 
(n.d.).

Aguilar, P., Lopez-Cobo, I., Cuadrado, F., y Benítez, I. (2019). Social and Emotional Competences in Spain: A Comparative Evaluation Between Spanish Needs and an International Framework Based on the Experiences of Researchers, Teachers, and Policymakers. Frontiers in Psychology, 10, 1-12. THHPS://DOI.ORG/10.3389/ fpsyg.2019.02127

Alemañy, C. (2009). La docencia: enfermedades frecuentes de esta profesión. Cuadernos de educación y desarrollo, 1(1), 1-5. Retrieved from https://bit.ly/2XQh92Y

Aliaga, J., Rodríguez, R., Ponce, C., Frisancho, A., y Enríquez, J. (2006). Escala de desesperanza de Beck (BHS): Adaptación y características psicométricas. Revista IIPSI, 9(1), 69-79. https://doi.org/10.15381/ rinvp.v9i1.4029

Alonso, F. (2014). Una panorámica de la salud mental de los profesores. Revista Iberoamericana de Educación, 66(1), 19-30. https://doi.org/10.35362/rie660375

Anastasi, A., y Urbina, S. (2008). Test Psicológicos. Prentice Hall. Retrieved from https://bit.ly/2BvcXyk Araujo, P. (2017). Habilidades sociales y desempeño docente en los institutos de Educación Superior Tecnológico de la Selva Central. Retrieved from https://bit.ly/3cZWAJ7

Arón, A., y Milicic, N. (2000). Desgaste profesional de los profesores y clima social escolar. Revista latinoamericana de psicología, 33(3), 447-466. Retrieved from https://bit.ly/2AQXV5q

Beck, A., y Steer, R. (2011). Manual. BAI. Inventario de Ansiedad de Beck [Adaptación española de J. Sanz]. Pearson.

Buela-Casal, G., y Sierra, J. C. (1997). Manual de Evaluación Psicológica. Fundamentos, técnicas y aplicaciones. Siglo XXI.

Caballo, V. (2000). Manual de evaluación y entrenamiento de las habilidades sociales. Siglo XXI.

Cano, F. J., Rodríguez, L., y García, J. (2007). Adaptación española del Inventario de Estrategias de Afrontamiento. Actas españolas de Psiquiatría, 35(1), 29-39. Retrieved from https://bit.ly/2MqFdnZ

Cardona, M. C., Chiner, E., y Lattur, M. A. (2011). Diagnóstico Psicopedagógico. Editorial Club Universitario.

Cardozo-Gutiérrez, L. (2016). El estrés en el profesorado. Revista de Investigación Psicológica, 15, 75-98.

Retrieved from https://bit.ly/3gPg5VL

Carrasco, I., Clemente, M., y Llavona, L. (1989). Análisis del inventario de aserción de Gambrill y Richey. Estudios de Psicología, 10(37), 63-74. https://doi.org/10.1080/02109395.1989.10821107

Cassullo, G. L., y García, L. (2015). Estudio de las Competencias Socio Emocionales y su Relación con el Afrontamiento en Futuros Profesores de Nivel Medio. Revista Electrónica Interuniversitaria de Formación del Profesorado, 18(1), 213-213. https://doi.org/10.6018/reifop.18.1.193041

Castaños, S., Reyes-Lagunes, I., Rivera, S., y Díaz-Loving, R. (2011). Estandarización del Inventario de Asertividad de Estandarización del Inventario de Asertividad de Gambrill y Richey-II. Revista Iberoamericana de Diagnóstico y Evaluación RIDEP, 29(1), 27-50. Retrieved from https://bit.ly/2XZNPY6

Céspedes, A. (2008). Educar las emociones, Educar para la vida. Ediciones B.

Charter, R. A. (1999). Sample Size Requirements for Precise Estimates of Reliability, Generalizability, and Validity Coefficients. Journal of Clinical and Experimental Neuropsychology, 21(4), 559-566. https://doi.org/ 10.1076/jcen.21.4.559.889

CIFE. (2018). Instrumento "Cuestionario de satisfacción con el instrumento". Centro Universitario CIFE. Retrieved from https://bit.ly/2Axrp8C

Cronbach, L. J. (1951). Coefficient alpha and the internal structure of tests. Psychometrika, 16(3), 297-334. https://doi.org/10.1007/bf02310555

Crosier, D., y Donkova, R. (2018). La salud mental en la educación: un problema silenciado en nuestro tiempo. Centro Nacional de Innovación e Investigación Educativa. . Retrieved from https: / / bit.ly/3gMt3Um

Cruz-Avelar, A., y Cruz-Peralta, E. (2017). Metodología para la construcción de instrumentos en salud. Pediátrica, 26(3), 100-105. Retrieved from https://bit.ly/3rm6w4Q

de-la Salud, O.-M. (2013). Plan de acción sobre salud mental 2013-2020. Retrieved from https:// bit.ly/30e3oOJ de-la Salud, O.-M. (2017). Salud mental. Temas de salud mental. Retrieved from https://bit.ly/2Xzx47c de México, G. (2013). Decreto por el que se reforman y adiciona diversas disposiciones de la Ley General de Salud, en materia de Salud mental. Retrieved from https://bit.ly/3cyhPiS

Donoso, S. (2018). Nuevo Rol del Docente, Nuevos Desafíos a la Docencia. Calidad en la Educación, 15, 1-11. https://doi.org/10.31619/caledu.n15.445

Elias, M. (2003). Aprendizaje académico y socio-emocional. International Academy of Education y Oficina Internacional de educación. Retrieved from https://bit.ly/2XvjvWj

Epstein, M. H. (2004). Escala de calificación conductual y emocional, segunda edición: un enfoque de evaluación basado en las fortalezas. Pro-Ed. 
Espinoza-Venegas, M., Sanhueza-Alvarado, O., Ramírez-Elizondo, N., y Sáenz-Carrillo, K. (2015). Validación de constructo y confiabilidad de la escala de inteligencia emocional en estudiantes de enfermería. Revista Latino-Americana de Enfermagem, 22(1), 139-147. https://doi.org/10.1590/0104-1169.3498.2535

Fernandez-Berrocal, P., Extremera, N., y Ramos, N. (2004). Validity and Reliability of the Spanish Modified Version of the Trait Meta-Mood Scale. Psychological Reports, 94(3), 751-755. https://doi.org/10.2466/pr0.94.3 $.751-755$

Fernández-Gómez, E., Martín-Salvador, A., Luque-Vara, T., Sánchez-Ojeda, M. A., Navarro-Prado, S., y Enrique-Mirón, C. (2020). Content Validation through Expert Judgement of an Instrument on the Nutritional Knowledge, Beliefs, and Habits of Pregnant Women. Nutrients, 12(4), 1136-1136. https://doi.org/10.3390/ nu12041136

Fragoso-Luzuriaga, R. (2015). Inteligencia y competencias emocionales en educación superior, ¿un mismo concepto? Revista Iberoamericana de Educación Superior, 6(16), 110-125. Retrieved from https://bit.ly/ $2 \mathrm{U} 6 \mathrm{U} 4 \mathrm{~s} 6$

García, B. (2018). Las habilidades socioemocionales, no cognitivas o "blandas": aproximaciones a su evaluación. Revista Digital Universitaria, 19(6), 1-17. https://doi.org/10.22201/codeic.16076079e.2018.v19n6.a5. García, J., y Vélez, C. (2017). Determinantes sociales de la salud y la calidad de vida en población adulta de Manizales, Colombia. Revista Cubana de Salud Pública, 43(2), 191-203. Retrieved from https://bit.ly/3chxbvv Gismero, E. (2010). Escala de Habilidades Sociales. Tea Ediciones.

González, L., y Espinoza, O. (2018). Calidad en la educación superior: concepto y modelos. Calidad en la educación, 28, 248-276. https://doi.org/10.31619/caledu.n28.210

González-Ortega, Y. (2008). Instrumento cuidado de comportamiento profesional: validez y confiabilidad. Aquichan, 8(2), 170-182. Retrieved from https://bit.ly/3gPUfl9

Gutiérrez-Torres, A. M., y Buitrago-Velandia, S. J. (2019). Las Habilidades Socioemocionales de los Docentes, herramientas de paz en la escuela. Praxis \& Saber, 10(24), 167-192. https://doi.org/10.19053/22160159.v10 .38.n25.2019.9819

Haynes, S. N., Richard, D. C. S., y Kubany, E. S. (1995). Content validity in psychological assessment: A functional approach to concepts and methods. Psychological Assessment, 7(3), 238-247. 10.1037/1040-3590.7 .3 .238

Hernández-Sampieri, R., Fernández, C., y Batista, P. (2003). Metodología de la investigación. México: McGrawHill.

Huerta, M. (2019). Evaluación de habilidades socioemocionales y transversales: un estado del arte. In UEALC: DIALOGAS, Adelante, Agcid Chile, MESACTS y CAF-banco de desarrollo de América Latina. Retrieved from https://bit.ly/2zarRco

Hyrkäs, K., Appelqvist-Schmidlechner, K., y Paunonen-IImonen, M. (2003). Translating and validating the Finnish version of the Manchester Clinical Supervision Scale. Scandinavian Journal of Caring Sciences, 17(4), 358-364. https://doi.org/10.1046/j.0283-9318.2003.00236.x

Jones, S. M., Bouffard, S. M., y Weissbourd, R. (2013). Educators' Social and Emotional Skills Vital to Learning. Phi Delta Kappan, 94(8), 62-65. https://doi.org/10.1177/003172171309400815

Juárez-Hernández, L. (2018). Manual práctico de estadística básica para la investigación. Kresearch Corp.

Juárez-Hernández, L., y Tobón, S. (2018). Análisis de los elementos implícitos en la validación de contenido de un instrumento de investigación. Espacios, 39(45), 23-30. Retrieved from https:// bit.ly/303IT8k

Kafka, T. (2016). A List of Non-Cognitive Assessment Instruments. Community Collage Research Center. Retrieved from https://bit.ly/3ITVBya

Kankaras, M., Renbarger, R., y Feron, E. (2019). Assessing students' social and emotional skills through triangulation of assessment methods. OECD Education Working Papers. OECD iLibrary, 208, 2-66. https:// doi.org/10.1787/19939019

Kankaras, M., y Suarez-Álvarez. (2019). Assessment framework of the OECD Study on Social and Emotional Skills. OECD Education Working Papers. OECD iLibrary, 207, 1-109. https://doi.org/10.1787/5007adef-en Kent, M. (2003). Diccionario Oxford de Medicina y Ciencias del Deporte. Editorial Paidotribo.

Kerlinger, F., y Howard, L. (2002). Investigación del comportamiento. Métodos de investigación en ciencias sociales. McGraw-Hill.

Koller, I., Levenson, M. R., y Glück, J. (2017). What Do You Think You Are Measuring? A Mixed-Methods Procedure for Assessing the Content Validity of Test Items and Theory-Based Scaling. Frontiers in Psychology, 8(126), 1-20. https://doi.org/10.3389/fpsyg.2017.00126

Koning, A. J., y Franses, H. B. (2003). Confidence intervals for Cronbach's alpha Coefficient values. ERIM Report Series Reference, No. ERS-041-MKT. Retrieved from https://bit.ly/3eLuDUx

Lagunes-Córdoba, R. (2017). Recomendaciones sobre los procedimientos de construcción y validación de instrumentos y escalas de medición en psicología de la salud. Psicológicas y salud, 27(1), 5-18. Retrieved from https://bit.ly/3ckU29q 
Leyva, Y. (2010). Una reseña sobre la validez de constructo de pruebas referidas a criterio. Perfiles Educativos, 36(131), 131-154. Retrieved from https://bit.ly/3cSKUG1

Llach, B. (2004). Qué es y cómo se mide la calidad de vida relacionada con la salud. Gastroenterología y Hepatología, 23(3), 2-6. Retrieved from https://bit.ly/395ZNFJ

Llorent, V., Zych, I., y Varo-Millán, J. C. (2020). Competencias socioemocionales autopercibidas en el profesorado universitario en España. Educación XX1, 23(1), 23-23. https://doi.org/10.5944/educxx1.23687

Llunch, M. T. (1999). Construcción de una escala para evaluar la salud mental positive. Retrieved from https://bit.ly/3dHADxl

López-Móndejar, L., y Tomás, P. (2017). Development of Socio-emotional Skills through Cooperative Learning in a University Environment. Procedia - Social and Behavioral Sciences, 237, 432-437. https://doi.org/10.1016/ j.sbspro.2017.02.086

Marenco-Escudero, A., y Ávila Toscano, J. (2016). Burnout y problemas de salud mental en docentes: diferencias según características demográficas y socio laborales. Psychologia. Avances de la disciplina, 19(1), 91-100. Retrieved from https://bit.ly/307tp2a

Martínez, J. (2014). Construcción y Validación de un Instrumento para Evaluar la Actitud hacia la Salud Mental. CLIDi, 1, 1-6. Retrieved from https://bit.ly/3gT2E7d

Martínez-Otero, V. (2003). Estrés y ansiedad en los docentes. Pulso: Revista de educación, 1(17), 9-21. Retrieved from https://bit.ly/2U5K3eu

Martín-Lesende, I. (2013). Escalas y pruebas de valoración funcional y cognitiva en el mayor. Actualización en Medicina Familiar, 9(9), 508-514. Retrieved from https://bit.ly/3lJxu5a

Mavrou, I. (2015). Análisis factorial exploratorio: cuestiones conceptuales y metodológicas. Revista Nebrija, 19, 71-80. https://doi.org/10.26378/rnlael019283

Mayer, J., y Salovey, P. (1997). What is emotional intelligence? In P. Salovey y D. J. Sluyter (Eds.), Emotional Development and Emotional Intelligence. Educational Implications (p. 3-34). Basic Books.

Mckenzie, D. (2014). Hard measurement of Soft Skills. World Bank. Retrieved from https://bit.ly/3tPJDbO

McKown, C. (2017). Social-Emotional Assessment, Performance, and Standards. The Future of Children, 27(1), 157-178. https://doi.org/10.1353/foc.2017.0008

Meliá, J. L. (2001). Teoría de la fiabilidad y la validez. Cristóbal Serrano.

Meliá, J. L., y Peiró, J. M. (1989). La medida de la satisfacción laboral en contextos organizacionales: el Cuestionario de satisfacción laboral S20/23. Psicologemas, 5, 59-74. Retrieved from https://bit.ly/2MqJ2cL

Meliá, J. L., y Peiró, J. M. (1998a). Cuestionario de satisfacción laboral S20/23. Psicología de la seguridad laboral-Universitat de Valencia. Retrieved from https://bit.ly/3eRV633

Meliá, J. L., y Peiró, J. M. (1998b). Factores del Cuestionario de satisfacción laboral S4/82. Psicología de la seguridad laboral-Universitat de Valencia. Retrieved from https://bit.ly/2XVMMbK

Mena, E., Isadora, M., Romagnoli, C., y Valdés, A. M. (2009). El impacto del desarrollo de habilidades socio afectivas y éticas en la escuela. Actualidades Investigativas en Educación, 9(3), 1-21. Retrieved from https://bit.ly/2Adg6SZ

Mendoza, R. L. (2010). A Cost-Benefit Analysis of the Legalization of an Informal Health Sector. Journal of Social Sciences, 6, 74-84. https://doi.org/10.3844/jssp.2010.74.84

mexicano-del Seguro-Social, I. (2018). Salud Mental. IMSS. Retrieved from https://bit.ly/2YbZLWX

Mikulic, I. M., y Crespi, M. (2008). Adaptación y validación del inventario de respuestas de afrontamiento de moos (CRI-A) para adultos. Anuario de investigaciones, 15, 305-312. Retrieved from https://bit.ly/2XXKhWI

Mikulic, I. M., Crespi, M., y Radusky, P. (2015). Construcción y validación del Inventario de Competencias Socioemocionales para Adultos (ICSE). Interdisciplinaria: Revista de Psicología y Ciencias Afines, 32(2), 307330. https://doi.org/10.16888/interd.2015.32.2.7

Mills, A., Butt, J., Maynard, I., y Harwood, C. (2012). Identifying factors perceived to influence the development of elite youth football academy players. Journal of Sports Sciences, 30(15), 1593-1604. https://doi.org/10.1080/ 02640414.2012.710753

Mingote-Adán, J. C., y Núñez-López, C. (2011). Importancia de la consideración de la salud mental en la gestión de la salud laboral: una responsabilidad compartida. Medicina y Seguridad del Trabajo, 57(1), 239262. https://doi.org/10.4321/s0465-546×2011000500015

Montero, I., y León, O. (2007). A guide for naming research studies in Psychology. Journal of Clinical and Health Psychology, 7(3), 847-862. Retrieved from https://bit.ly/2A3xbPb

Morán, V. E., y Olaz, F. O. (2014). Instrumentos de evaluación de habilidades sociales en América Latina: un análisis bibliométrico. Revista de Psicología, 23(1), 93-93. https://doi.org/10.5354/0719-0581.2014.32877

Moss, R. (2010). CRI-A, Inventario de Repuestas de Afrontamiento para Adultos. Manual. España, TEA ediciones: Tea Ediciones.

Naciones-Unidas. (2015). Transformar nuestro mundo: la Agenda 2030 para el Desarrollo Sostenible. Retrieved from https://bit.ly/3eQINUE 
Navarro-Prado, S., Sánchez-Ojeda, M. A., Martín-Salvador, A., Luque-Vara, T., Fernández-Gómez, E., y Caro-Morán, E. (2020). Development and Validation of a Rating Scale of Pain Expression during Childbirth (ESVADOPA). International Journal of Environmental Research and Public Health, 17(16), 5826-5826. https:// doi.org/10.3390/ijerph17165826

Núñez-Hernández, C. E., del Salto, V. S. H., Jerez-Camino, D. S., Rivera-Flores, D. G., y NúñezEspinoza, M. W. (2018). Las habilidades sociales en el rendimiento académico en adolescentes. Revista de Comunicación de la SEECl, 47, 37-37. https://doi.org/10.15198/seeci.2018.0.37-49

Nunnally, J., y Bernstein, I. (1994). Psychometric Theory. McGraw-Hill.

OECD. (2015). Skills for Social Progress. The power of social and emotional skills. https://doi.org/10.1787/ 9789264226159-en

OECD. (2019). OECD Study on Social and Emotional Skills. How we measure social and emotional skills. Retrieved from https://bit.ly/3cd0VcS

Ornelas, A., y Olivia, A. (2017). Salud mental y calidad de vida: Su relación en los grupos etarios. PSIENCIA. Revista Latinoamericana de Ciencia Psicológica, 9(2), 1-16. Retrieved from https://bit.ly/372wphn

Otero, M., Cresta, C., Finoli, M., y País, E. (2017). Evaluación de habilidades socioemocionales. UEICEE. Retrieved from https://bit.ly/39abCee

Palomera, R., Fernández-Berrocal, P., y Brackett, M. (2008). La inteligencia emocional como una competencia básica en la formación inicial de los docentes: algunas evidencias. Psychology, 15(6), 437-454. Retrieved from https:// bit.ly/2AFw7kq

Panizza, M. (2015). Evaluación de habilidades socioemocionales a partir de la prueba PISA. Instituto Nacional de Evaluación Educativa. Retrieved from https://bit.ly/3vSI5PP

Papieska, J., Spilt, J. L., Roorda, D. L., y Laevers, F. (2019). Promoting socioemotional competence in primary school classrooms: Intervention effects of the EMOscope. European Journal of Developmental Psychology, 16(1), 97-112. https://doi.org/10.1080/17405629.2017.1342620

Penfield, R. D., y Peter R. Giacobbi, J. (2004). Applying a Score Confidence Interval to Aiken's Item ContentRelevance Index. Measurement in Physical Education and Exercise Science, 8(4), 213-225. https://doi.org/ 10.1207/s15327841mpee0804_3

Pérez-Escoda, N., Bisquerra, R., Filella, G., y Soldevila, A. (2010). Construcción del Cuestionario de Desarrollo Emocional de Adultos (QDE=A). Revista Española de Orientación y Psicopedagogía, 21(2), 367-379. https:// doi.org/10.5944/reop.vol.21.num.2.2010.11539

Pierobon, A. (2020). Cuestionarios auto-reportados para la evaluación de la funcionalidad en pacientes de consultorio externo: herramientas disponibles para la población argentina. Argentinian Journal of Respiratory and Physical Therapy, 1(3), 1-3. Retrieved from https://bit.ly/3sfUN97

Plutchik, R., van Praag, H. M., Conte, H. R., y Picard, S. (1989). Correlates of suicide and violence risk 1: The suicide risk measure. Comprehensive Psychiatry, 30(4), 296-302. https://doi.org/10.1016/0010-440x(89)90053 $-9$

Pública, S. D. E. (2018). Retrieved from https://bit.ly/2Mw1W26

Rikoon, S., Brenneman, M., Petway, I. I., y K. (2016). Assessing Social-Emotional Learning. National Association of State Boards of Education, 20-23. Retrieved from https://bit.ly/39bKMSX

Rodríguez-Bustamante, A., López-Arboleda, G. M., y Echeverri-Álvarez, J. C. (2017). El aula de paz: familia y escuela en la Construcción de una cultura de paz en Colombia. Perseitas, 5(1), 206-206. https://doi.org/ $10.21501 / 23461780.2243$

Rubio, G., Montero, I., Jáuregui, J., Villanueva, R., Casado, M., Marín, J. J., y Santo-Domingo, J. (1998). Validación de la escala de riesgo suicida de Plutchik en población española. Archivos de Neurobiología, 61(2), 143-152.

Ruíz, C. (2002). Instrumentos de Investigación Educativa. Procedimientos para su Diseño y Validación. CA: Tipografía Litografía Horizontes.

Sanz, J. (2014). Recomendaciones para la utilización de la adaptación española del Inventario de Ansiedad de Beck (BAI) en la práctica clínica. Clínica y Salud, 25(1), 39-48. https://doi.org/10.5093/cl2014a3

Sanz, J., y Navarro, M. E. (2003). Propiedades psicométricas de una versión española del inventario de ansiedad de Beck (BAI) en estudiantes universitarios. Ansiedad y Estrés, 9(1), 59-84. Retrieved from https:// bit.ly/36YHKz2

Sanz, J., Perdigón, A. L., y Vázquez, C. (2003). Adaptación española del Inventario para la Depresión de BeckII (BDI-II): 2. Propiedades psicométricas en población general. Clínica y Salud, 14(3), 249-280. Retrieved from https://bit.ly/3dzEGMi

Schonert-Reichl, K. A. (2017). Social and Emotional Learning and Teachers. The Future of Children, 27(1), 137-155. https://doi.org/10.1353/foc.2017.0007

Tassé, M. J., Schalock, R. L., Balboni, G., Bersani, H., Borthwick-Duffy, S. A., Spreat, S., Thissen, D., Widaman, K. F., y Zhang, D. (2021). TEA Ediciones y HOGREFE. In DABS, Escala de Diagnóstico de Conducta 
adaptativa . TEA Ediciones y HOGREFE. Retrieved from https://bit.ly/3rhBCdZ

Uribe, J. F. (2006). La escala mexicana de desgaste ocupacional (EMEDO): estudio exploratorio de un instrumento de burnout para mexicanos. In Primer Foro de las Américas en investigación sobre factores psicosociales, estrés y salud mental en el trabajo. Retrieved from https://bit.ly/2Byu9D6

Uribe, J. F. (2007). Estudio confirmatorio de la escala mexicana de desgaste ocupacional (EMEDO): un instrumento de burnout para mexicanos. Revista Interamericana de Psicología Ocupacional, 26(1), 7-21. Retrieved from https://bit.ly/3eMxYTx

Velarde-Jurado, E., y Avila-Figueroa, C. (2002). Evaluación de la calidad de vida. Salud Pública de México, 44(4), 349-361. https://doi.org/10.1787/1993901910.1590/s0036-36342002000400009

Vélez, C., Vidarte, J. A., Arango, A., Patiño, B. S., y Rondón, Y. A. (2021). Adaptation and validation of content of the sedentary behavior questionnaire. Hacia la Promoción de la Salud, 26(1), 141-155. https://doi.org/10.1787/ 1993901910.17151/hpsal.2021.26.1.12

Vilagut, G., Ferrer, M., Rajmil, L., Rebollo, P., Permanyer-Miralda, G., Quintana, J. M., Santed, R., Valderas, J. M., Ribera, A., Domingo-Salvany, A., y Alonso, J. (2005). El Cuestionario de Salud SF-36 español: una década de experiencia y nuevos desarrollos. Gaceta Sanitaria, 19(2), 135-150. https://doi.org/10.1787/ $1993901910.1157 / 13074369$

Welch, S., y Comer, J. (2001). Quantitative Methods for Public Administration: Techniques and Applications. Dorsey Press.

Whitehead, J., y Suave, J. (2018). The Importance of Teacher Well-being and Social and Emotional Learning: A Literature Review Prepared for Millennium.org. Retrieved from https://bit.ly/3vRtTGV

Wilson-Ahlstrom, y Yohalem, N. (2014). From Soft Skills to Hard Data: Measuring youth program outcomes. The Forum for Youth Investment. Retrieved from https://bit.ly/3sfUtaF 